\title{
Epidemiology of Burn Cases in Erbil Governorate
}

\author{
Pakestan Hamadamin Yousif ${ }^{1}$, Hoshyar Amin Ahmed ${ }^{2}$ \\ ${ }^{1}$ M.Sc., Department of Nursing, Soran Technical Institute, Erbil Polytechnic University, Kurdistan Region, Iraq, \\ ${ }^{2}$ Assist. Prof. Dr. Dean of Erbil Health Technical College, Erbil Polytechnic University, Iraq
}

\begin{abstract}
Background and Objectives: Management and prevention of burns are done through epidemiological study. The mortality rate of burn injuries is seven times higher in the low income countries than the high income countries. The aim of this research was to study the epidemiology of burn injury cases through analysis of the cause, magnitude and profile of burn in Erbil governorate as a method for planning preventive and management strategies.
\end{abstract}

Method: A retrospective study was conducted on 593 burn cases to assess the epidemiology of burn injury cases in Erbil Governorate the capital of Iraqi Kurdistan Region from 2012 to the end of 2016. Descriptive and inferential statistics were used through the Microsoft Excel Database and the Statistical Package for Social Sciences (SPSS, Version 24). The P value of $>0.05$ was considered as non-statistically significant. The $P$ value of $\leq 0.05$ was considered as statistically significant. The $P$ value of $\leq 0.01$ was considered as highly statistically significant.

Results: The total number of burn cases in Erbil governorate during the period 2012 to 2016 was 593 victims. The mean $( \pm \mathrm{SD})$ age of the studied cases was $26 \pm 10.9$ years ranging from one to 82 years. The highest number $189(31.9 \%)$ of registered burn cases was in 2013, while the lowest number $74(12.5 \%)$ of cases was in 2015. The female to male ratio of the burn victims was $2.1: 1$. More than half $(56.7 \%)$ of them were married, while the others (43.3\%) were single. Husband and polygamy were the highest leading causes of the burn cases in a way that each of the causes had formed $18.4 \%$ of the cases. The highest number $(44.35 \%)$ of the burn cases was among the age group 21 to 30 years. There was a significant correlation between the marital status of the studied cases with the causes behind the burn injuries at $p=0.027$ and highly significant correlation of the marital status with the survival rates in. The correlation was highly significant between the gender of the studied samples and the cause of the burns. There was no significant correlation between the educational levels of the burn victims with the causes and the survival rates as well.

Key words: immortal, inflicted, burn, injury, Erbil.

\section{Introduction}

Study of the cause and the history of burn in any population can be done through an epidemiological research which is considered as the first step of management and prevention of burns ${ }^{10}$. The socioeconomic stability of any country is an influential

\section{Corresponding Author:}

Hoshyar Amin Ahmed

Assist. Prof. Dr. Dean of Erbil Health Technical

College Erbil Polytechnic University, Iraq;

E-mail: hoshyar@epu.edu.iq factor for burn profiles ${ }^{12}$. About 11 million people were burnt severely in 2014 worldwide. All these cases required medical care. As a public health problem, annually burns lead to about 180000 deaths. Most of cases occur in the middle and low income countries including South East Asian and African regions. Burn death rates are decreasing in the high-income countries. This rate in the middle and low income countries is seven times higher than the higher income countries. Furthermore, the non-fatal burning injuries are considered as a leading cause for morbidity which includes disfigurement and disability which may result in stigma with rejection and prolonged hospitalization. Although burns are 
preventable but mainly it occurs at the workplaces and homes (WHO, 2017). Burns treatment had improved from the primarily topical therapy including the strange and wonderful topical mixtures, concoctions consisting of cow dung, bees wax, ram's horn and barley porridge with resin-socked dressing of the tree shrub acacia with red ochre, and copper in ancient times to the role of joint scientific fields of topical therapy, antibiotics, fluid resuscitation, skin excision and grafting, respiratory and metabolic care and nutrition. The majority of the advances in burn management had occurred in the last 50 years, spurred on by wars and great fires ${ }^{1}$. The use of systemic antibiotics and topical silver therapy greatly reduced sepsis related mortality. These scientific changes jointly with the advent of antiseptic surgical techniques, burn depth classification and skin grafting allowed the excision and coverage of full-thickness burns which resulted in greatly improved survival rates. Advances in the methods of assessing the surface area of burns helped for more accurate fluid resuscitation, minimizing the effects of shock and avoiding fluid overloading. The metabolic care, nutritional support and care of inhalational injuries further improved the outcome of burn patients ${ }^{13}$.

\section{Subjects and Methods}

A retrospective study was conducted on 593 burn cases to assess the epidemiology of burn injury cases in Erbil Governorate the capital of Iraqi Kurdistan Region from 2012 to the end of 2016. The inclusion criteria for the study sample were the burn cases that had been attended to the law department of the hospitals and police stations. Descriptive and inferential statistics were used through the Microsoft Excel Database jointly with the Statistical Package for Social Sciences (SPSS, Version 24). The $P$ value of $>0.05$ was considered as non-statistically significant. The $\mathrm{P}$ value of $\leq 0.05$ was considered as statistically significant. The $\mathrm{P}$ value of $\leq$ 0.01 was considered as highly statistically significant.

\section{Results}

The total number of burn cases in Erbil governorate during the period 2012 to 2016 was 593 victims. The mean $( \pm \mathrm{SD})$ age of the studied cases was $26 \pm 10.9$ years ranging from one to 82 years.

Table 1 show that the highest number 189 (31.9\%) of registered burn cases was in 2013, while the lowest number 74 (12.5\%) of cases was in 2015 .

Table 1: Number of burn cases per year

\begin{tabular}{|l|l|l|}
\hline Year & F & $\mathbf{\%}$ \\
\hline 2012 & 155 & 26.1 \\
\hline 2013 & 189 & 31.9 \\
\hline 2014 & 97 & 16.4 \\
\hline 2015 & 74 & 12.5 \\
\hline 2016 & 78 & 13.2 \\
\hline Total & 593 & 100 \\
\hline
\end{tabular}

Table 2 shows the socio-demographic characteristics of the studied burn cases. About two thirds $(67.8 \%)$ of them were females and about one third (32.2\%) of them were males. The female to male ratio of the burn victims was $2.1: 1$. More than half $(56.7 \%)$ of them were married, while the others $(43.3 \%)$ were single. The highest percentage $(54.5 \%)$ of the victims were primary school graduates. The illiterates, intermediate school graduates and high school graduates had formed 19.9\%, 23.8\%, and $1.8 \%$ of the studied cases respectively.

Table 2: The socio-demographic characteristics of the sample

\begin{tabular}{|l|l|l|}
\hline Characteristics of the sample & Frequency (F) & Percentage (\%) \\
\hline Gender & 191 & 32.2 \\
\hline Male & 402 & 67.8 \\
\hline Female & \multicolumn{2}{|l|}{} \\
\hline Marital status & \multicolumn{2}{|l|}{} \\
\hline
\end{tabular}


Cont... Table 2: The socio-demographic characteristics of the sample

\begin{tabular}{|l|l|l|}
\hline Married & 336 & 56.7 \\
\hline Single & 257 & 43.3 \\
\hline Education & \multicolumn{2}{|l|}{} \\
\hline Illiterate & 118 & 19.9 \\
\hline Primary school & 323 & 54.5 \\
\hline Intermediate school & 141 & 23.8 \\
\hline High school & 11 & 1.8 \\
\hline Total & 593 & 100 \\
\hline
\end{tabular}

Table 3 reveals the overall causes of the burn cases during the year 2012 to 2016. Husband and polygamy were the highest leading causes of the burn cases in a way that each of the causes had formed $18.4 \%$ of the cases. Interference of parents in law had formed only $1.2 \%$ of the leading causes of burn cases.

\section{Table 3: Causes of burn cases}

\begin{tabular}{|l|l|l|}
\hline Cause & Frequency & \% \\
\hline Husband & 109 & 18.4 \\
\hline Forced marriage & 62 & 10.5 \\
\hline Psychiatric disease & 82 & 13.8 \\
\hline Bad hospital treatment & 59 & 9.9 \\
\hline Premature marriage & 83 & 14 \\
\hline Interference of parents in law & 7 & 1.2 \\
\hline Polygamy & 109 & 18.4 \\
\hline Unknown & 25 & 4.2 \\
\hline Accident & 57 & 9.6 \\
\hline Total & 593 & 100 \\
\hline
\end{tabular}

Table 4 reveals that the highest number (44.35\%) of the burn cases was among the age group 21 to 30 years and the lowest number of cases $(1.01 \%)$ was among victims with $>60$ years of age.

Table 4: Distribution of the burn cases by their age

\begin{tabular}{|l|l|l|}
\hline Age / years & F & \% \\
\hline $1-10$ & 14 & 2.36 \\
\hline $11-20$ & 175 & 29.51 \\
\hline $21-30$ & 263 & 44.35 \\
\hline $31-40$ & 85 & 14.33 \\
\hline $41-50$ & 36 & 6.07 \\
\hline $51-60$ & 14 & 2.36 \\
\hline$>60$ & 6 & 1.01 \\
\hline Total & 593 & 100 \\
\hline
\end{tabular}


Table 5 indicates the burn size percentages of the studied sample. Unfortunately, the highest number (21.92) of the burn cases had suffered from $96 \%$ to $100 \%$ of burn size and the lowest number (1.01) of cases suffered from $31 \%-35 \%$ of burn size.

Table 5: The burn sizes by percentage

\begin{tabular}{|l|l|l|}
\hline Burn size \% & F & \% \\
\hline $5-10$ & 32 & 5.40 \\
\hline $11-15$ & 53 & 8.94 \\
\hline $16-20$ & 47 & 7.93 \\
\hline $21-25$ & 49 & 8.26 \\
\hline $26-30$ & 53 & 8.94 \\
\hline $31-35$ & 6 & 1.01 \\
\hline $36-40$ & 8 & 1.35 \\
\hline $41-45$ & 15 & 2.53 \\
\hline $46-50$ & 7 & 1.18 \\
\hline $51-55$ & 7 & 1.18 \\
\hline $56-60$ & 41 & 6.91 \\
\hline $61-65$ & 10 & 1.69 \\
\hline $66-70$ & 37 & 6.24 \\
\hline $71-75$ & 9 & 1.52 \\
\hline $76-80$ & 30 & 5.06 \\
\hline $81-85$ & 16 & 2.70 \\
\hline $86-90$ & 44 & 7.42 \\
\hline $91-95$ & 13 & 2.19 \\
\hline $96-100$ & 130 & 21.92 \\
\hline Total & 593 & 100 \\
\hline & & \\
\hline
\end{tabular}

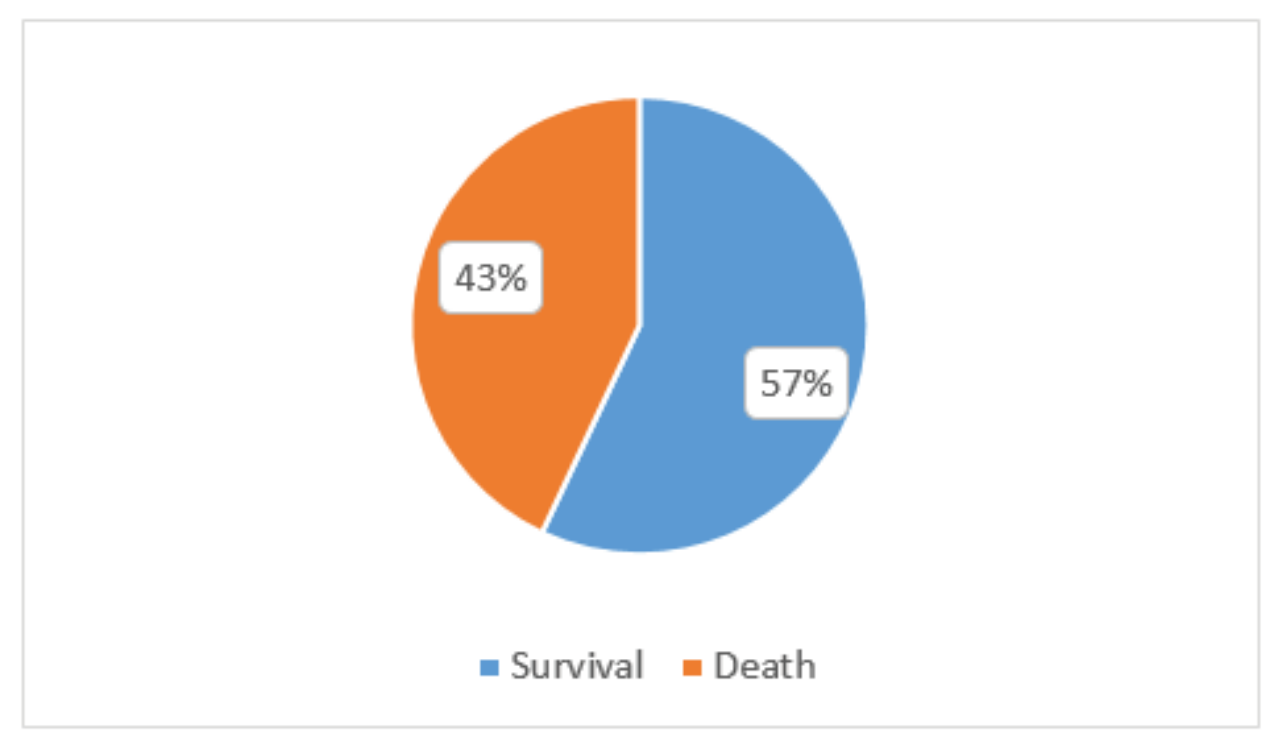

Figure 1. Proportion of survival and death of the burn injury cases

Figure 1 shows the proportion of survival and death of the burn injury cases. 
The correlational statistical findings of this study had been calculated. There was a significant correlation between the marital status of the studied cases with the causes behind the burn injuries at $p=0.027$ in one hand and highly significant correlation with the survival rates in another hand. The correlation was highly significant between the gender of the studied samples and the cause of the burns. There was no significant correlation between the educational levels of the burn victims with the causes and the survival rates as well.

\section{Discussion}

This study has analyzed the burn profiles in Erbil City from 2012 to 2016. The gradual decrease of recorded annual burn cases can be attributed to the shifting from kerosene use to other resources such as electrical heating systems in the study region. In a study by ${ }^{10}$ in Delhi, it has been concluded that economic uplift and shift from kerosene to safer LPG stoves has decreased the annual burn admission by $43 \%$ in a major burn unit of Delhi. The survival rate among all the burn cases during the 5 years was $46 \%$ and the death rate was $56 \%$. An Indian study by ${ }^{9}$ has reported $54.51 \%$ of death among the burn injury cases. They concidered the high mortality rate amongst burn patients as a major concern of health care towards burn injuries. The ratio of female to male among the currently studied cases was 2.1. Previous studies indicated that immolation and self burning is more common among young females than males in the Eastern Mediterranean region and South and Central Asia including Kurdistan Region. This phenomenon can be contributed to the social and psychological distresses arising from the male dominated society. Marriage is one of the common sources of conflict between genders and generations ${ }^{9}$. Another study in Iran by ${ }^{1}$ indicated the higher number of self-immolation victims among females than males. The current study revealed that burn cases had been occurred mostly among primary school graduates. This result came in coincide with that of Ahmadi et al., $2014{ }^{1}$ among the northern people of Iran which showed higher incidence of the self immollation among primary school graduates. In regard to the causes behind the self-immolation, it has beeen found that the social and psychiatric causes had formed the higher proportions than the bad hospital treatment and the accidental burn cases. Suicidal self-immolation is a terrible common method in Eastern societies. One study explored the leading causes to self-immolation in young Kurdish females. The latter study ralated the self-immolation attemts to absence of self-control over the personal life, family conflicts, to self-immolation attempts, including not having control over personal life, marital conflicts, looking for attention, seeking attention, feeling of guilt, and male domination among such communities ${ }^{8}$. Another study in Musil Teaching Hospital revealed that the self-inflicted burn cases had a significantly larger percentage of surface body area and this type of burning was the most common cause of hospital admissions among the burn cases which lead to the higher mortality in this patient group ${ }^{7}$. In a study on the syrian society, lower self-esteem, less life satisfaction, less marital satisfaction and more mental health symptomatology than monogamous marriages had been recorded. Many of the mental health symptoms were arised such as somatization, depression, hostility and psychoticism. Moreover, the latter wives reported less psychosocial problems than the second and first wives ${ }^{6}$. Premature marriage was one of the leading causes to self-immolation among the studied population of the current study. In many countries of the world, premature marriage before the age of 18 is a reality. This type of marriage is permitted according to the Universal Declaration of Human Rights for many young women who are encouraged by their families while they are still children aiming to get the financial and social benefits, although child marriage is considered as violation of human rights. Probably this will lead to early pregnancy and social isolation. Furthermore, it will lead to decrease the education ooportunity and poor training that will cause the gendered nature of poverty. The self-inflicted burn cases which had been detected by this study were resulted from the forced and/or the premature marriage that had been associated with the above mentioned sociocultural and economical factors. Although, nearly one in 10 burn cases of the present study had been recorded as result of the bad hospital treatment, but it has not been medically supported. In a study from Iran, the risk factors for death of the burned patient had been attributed to the age of the patients, the gender, and the size of the burn. Directing more attention to the vulnerable patients had been required to reduce the mortality rate and improvement of the patient survival ${ }^{11}$.

In regrd to the age of the most vulnerable age groups to burn injuries, the highest proportion of the 
victims were within the young age group of 21-30 years. This situation differs from one to another country or culture. In our study, the mean age \pm SD was $26 \pm 10.9$. According to a study in the United States of America the self-inflicted burn cases formed only $1 \%$ of the injury cases during 2011-2015. In Tabriz, Iran, among the 412 self-inflicted burns, the average age was 25.5 years and the majority $(99 \%)$ of the cases were female. About two thirds (76.5\%) were in the age groups of 15-19 and 20-29 years (Maghsoudi et al., 2004). In Tehran, Iran from 1997 to 1999, and among the 110 self-immolation patients, the mean age was 25 years.

\section{Conclusion}

This study revealed that number of burn cases had been decreased from 2012 to 2016. Females burn cases was higher than males by two folds. More than half of the cases was among the primary school graduates. Spouse, polygamy, premature marriage and psychological diseases were the top risk factors of selfinflicted burns. The highest percentage of burn cases was associated with the larger sizes of the burn injuries. Legal, social, and educational efforts are needed to decrease the accidental and self-immortal burns among the community members.

Financial Disclosure: There is no financial disclosure.

Conflict of Interest: None to declare.

Ethical Clearance: All experimental protocols were approved under the Department of Nursing and all experiments were carried out in accordance with approved guidelines.

\section{References}

1. Ahmadi M. Ranjbaran H. Azadbakht M. Gorji M H. \& Gorji A H. A survey of characteristics of selfimmolation in the northern Iran. Annals of medical and health sciences research. 2014; 4(3): 228-232.

2. icide and self-injury by burns in the Kurdistan Region of Iraq. The Sociological Review. 62(2); 237-254.

3. Ryan C M. Lee A. Kazis L E. Schneider J C. Shapiro G D. Sheridan R L. Reilly D. Recovery trajectories after burn injury in young adults: does burn size matter? Journal of Burn Care \& Research. 2015;36(1): 118-129.

4. Shabila N. Ismail K. \& Faidulla R. A four-year review of burn injuries in Emergency Management centre in Erbil, Iraqi Kurdistan. Zanco J Med Sci, 12. 2008; 111-117.

5. UNICEF. Early marriage a harmful traditional practice a statisAhuja R B. Bhattacharya S. \& Rai A. 2005. Changing trends of an endemic trauma. Burns. 2009; 35(5): 650-656.

6. Al-Krenawi A. Mental health and polygamy: The Syrian case. World journal of psychiatry. 2013; 3(1): 1 .

7. Al-Zacko S. Self-inflicted burns in Mosul: a crosssectional study. Annals of burns and fire disasters. 2012; 25(3): 121.

8. Amin P M. \& Mirlashari J. A cry for help and protest: self-immolation in young Kurdish Iraqi women-a qualitative study. International journal of community based nursing and midwifery. 2018; 6(1): 56.

9. Bhansali C A. Gandhi G. Sahastrabudhe P. \& Panse $\mathrm{N}$. Epidemiological study of burn injuries and its mortality risk factors in a tertiary care hospital. Indian Journal of Burns. 2017; 25(1): 62.

10. Bhattacharya S. Burn epidemiology-an Indian perspective. 2009.

11. Fazeli S. Karami-Matin R. Kakaei N. Pourghorban S. Safari-Faramani R. \& Safari-Faramani B. Predictive factors of mortality in burn patients. Trauma monthly. 2014; 19(1).

12. Forjuoh S N. Burns in low-and middle-income countries: a review of available literature on descriptive epidemiology, risk factors, treatment, and prevention. Burns. 2006; 32(5): 529-537.

13. Lee K C. Joory K. \& Moiemen N S. History of burns: the past, present and the future. Burns \& trauma. 2014; 2(4): 169.

14. Maghsoudi H. Garadagi A. Jafary G A. Azarmir G. Aali N. Karimian B. \& Tabrizi M. Women victims of self-inflicted burns in Tabriz, Iran. Burns. 2004; 30(3): 217-220.

15. Malic C. Karoo R. Austin O. \& Phipps A. Burns inflicted by self or by others - an 11 year snapshot. Burns. 2007; 33(1): 92-97. 
16. Mushin O P. Esquenazi M D. Ayazi S. Craig C. \& Bell D E. Self-inflicted burn injuries: Etiologies, risk factors and impact on institutional resources. Burns. 2019; 45(1): 213-219.
17. Othman N. Epidemiology of burn injuries in Sulaymaniyah province of Iraq. University of Nottingham. 2010. 